\title{
A combined fit of energy spectrum, shower depth distribution and arrival directions to constrain astrophysical models of UHECR sources
}

\section{Teresa Bister ${ }^{a, *}$ on behalf of the Pierre Auger ${ }^{b}$ Collaboration}

(a complete list of authors can be found at the end of the proceedings)

${ }^{a}$ Physics Institute IIIA, RWTH Aachen University, Otto-Blumenthal-Str., 52074 Aachen, Germany

${ }^{b}$ Observatorio Pierre Auger, Av. San Martín Norte 304, 5613 Malargüe, Argentina

E-mail: spokespersons@auger.org

The combined fit of the measured energy spectrum and distribution of depths of shower maximum of ultra-high-energy cosmic rays is known to constrain the parameters of astrophysical scenarios with homogeneous source distributions. Further measurements show that the cosmic-ray arrival directions agree better with the directions and fluxes of catalogs of starburst galaxies and active galactic nuclei than with isotropy.

Here, we present a novel combination of both analyses. For that, a three-dimensional universe model containing a nearby source population and a homogeneous background source distribution is built, and its parameters are adapted using a combined fit of the energy spectrum, depth of shower maximum distribution and energy-dependent arrival directions. The model takes into account a symmetric magnetic field blurring, source evolution and interactions during propagation.

We use simulated data, which resemble measurements of the Pierre Auger Observatory, to evaluate the method's sensitivity. With this, we are able to verify that the source parameters as well as the fraction of events from the nearby source population and the size of the magnetic field blurring are determined correctly, and that the data is described by the fitted model including the catalog sources with their respective fluxes and three-dimensional positions. We demonstrate that by combining all three measurements we reach the sensitivity necessary to discriminate between the catalogs of starburst galaxies and active galactic nuclei.

$37^{\text {th }}$ International Cosmic Ray Conference (ICRC 2021)

July 12th - 23rd, 2021

Online - Berlin, Germany

\footnotetext{
${ }^{*}$ Presenter
} 


\section{Introduction}

At the Pierre Auger Observatory, cosmic rays (CRs) with energies up to $O(100) \mathrm{EeV}$ can be detected and their properties can be measured, including energy, depth of the shower maximum $X_{\max }$ and arrival direction. The cosmic ray energy can be determined with the 1660 surface detector (SD) stations, which are distributed over an area of $3000 \mathrm{~km}^{2}$ and which detect extensive air showers produced by the primary CR during interactions with the atmosphere. For some events, $X_{\max }$ can be measured with the fluorescence detector (FD) on dark, cloudless nights. The charge of the primary particle cannot be measured directly, but $X_{\max }$ is related to it. Both measurements have been combined in [1] to fit a model containing a homogeneous distribution of sources to the data, and to gain knowledge about parameters of the sources of ultra-high-energy CRs. The identification of sources that are powerful enough to accelerate CRs to the highest energies is one of the prime goals of the Pierre Auger Observatory. Here, we demonstrate that by also using the arrival directions as a third observable in the fit, we can additionally model the energy-dependent contribution of a catalog of specific foreground sources.

These foreground sources are based on the findings in [2, 3], where the Pierre Auger Collaboration demonstrated that the measured arrival directions (ADs) agree better with catalogs of starburst galaxies (SBGs) or gamma-ray emitting active galactic nuclei (AGNs) than with isotropy. Currently, the correlation is detected at the $4.0 \sigma$ and $3.1 \sigma$ confidence levels [4], respectively, but differentiation between the two source classes remains challenging as the strongest sources of both catalogs are located at very similar directions. That analysis uses only the arrival directions $\vec{v}_{i}$ to

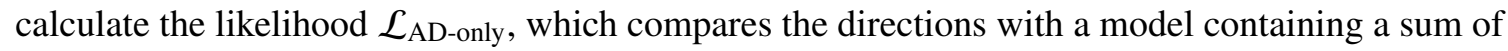
an isotropic component $B$, which follows the observatory exposure, and an anisotropic component $S$, which consists of Fisher distributions centered on each source, multiplied with a flux weight and accounting for the attenuation and the observatory exposure [2]:

$$
\log \mathcal{L}_{\mathrm{AD}-\text { only }}(f, \delta)=\sum_{i} \log \left(f \cdot S_{\delta}\left(\vec{v}_{i}\right)+(1-f) B\left(\vec{v}_{i}\right)\right):=\sum_{i} \log \left(\operatorname{pdf}_{\mathrm{AD}-\text { only }}\left(\vec{v}_{i}\right)\right)
$$

The model has two fit parameters, the size of the symmetric magnetic field smearing $\delta=\delta_{\mathrm{AD} \text {-only }}$ and the signal fraction $f=f_{\mathrm{AD} \text {-only }}$ which weights the isotropic and anisotropic contributions. Both parameters are determined by maximizing a likelihood ratio, the test statistic $\mathrm{TS}_{\mathrm{AD}}$ :

$$
\mathrm{TS}_{\mathrm{AD} \text {-only }}=2 \log \left(\mathcal{L}\left(f_{\mathrm{AD}-\text { only }}, \delta_{\mathrm{AD}-\text { only }}\right) / \mathcal{L}\left(f_{\mathrm{AD} \text {-only }}=0\right)\right)
$$

The ratio is calculated for several energy thresholds and the best-fit values for $f$ and $\delta$ are determined independently for each threshold. Currently, the highest TS $=24.8$ is reached for $f_{\mathrm{AD} \text {-only }} \simeq 9 \%$ and $\delta_{\mathrm{AD} \text {-only }} \simeq 15^{\circ}$ for an energy threshold of $38 \mathrm{EeV} \mathrm{[4]} \mathrm{for} \mathrm{the} \mathrm{SBG} \mathrm{catalog.}$

\section{Universe model}

In the following, we will present a combined fit which includes not only the energies and depths of shower maximum of the CRs, but also the energy-dependent arrival directions. For that, we will first introduce the universe model that can be fit to the data. 
We model the nearby universe as a sum of two parts, the foreground sources which are given by a catalog of either 44 starburst galaxies or 23 active galactic nuclei as in [4], and a background accounting for further unknown sources of the same type. All sources accelerate CRs to a maximum rigidity $R_{\text {cut }}$, so that the injected flux $J_{\text {inj }}$ follows a power-law with a broken exponential cutoff:

$$
J_{\text {inj }}\left(E_{\text {inj }}, A_{\text {inj }}\right)=J_{0} \cdot a_{i}\left(A_{\text {inj }}\right)\left(\frac{E_{\text {inj }}}{10^{18} \mathrm{eV}}\right)^{-\gamma} \begin{cases}1 & Z_{\text {inj }} R_{\text {cut }}<E_{\text {inj }} \\ \exp \left(1-\frac{E_{\text {inj }}}{Z_{\text {inj }} R_{\text {cut }}}\right) & Z_{\text {inj }} R_{\text {cut }} \geq E_{\text {inj }}\end{cases}
$$

Here, $a_{i}\left(A_{\text {inj }}\right)$ is the fraction of the element with mass number $A$ defined below the cutoff, $E_{\text {inj }}$ is the energy and $J_{0}$ is a normalization.

Since the goal of this method is a quickly adaptable model, we use 1-dimensional CRPropa3 simulations to account for the propagation between each source and Earth [5]. We produce a simulation database, consisting of $10^{6} \mathrm{CRs}$ for each energy bin $\left(10^{18.00} \mathrm{eV}, 10^{18.02} \mathrm{eV} \ldots 10^{21.00} \mathrm{eV}\right)$ for each representative element $(\mathrm{H}, \mathrm{He}, \mathrm{N}, \mathrm{Si}, \mathrm{Fe})$ and each distance, binned logarithmically between $1 \mathrm{Mpc}$ and $5700 \mathrm{Mpc}$ (redshift $z \approx 2$ ) in 118 bins. We use the Gilmore model [6] for the extragalactic background light and the TALYS model [7] for the photodisintegration, which corresponds to the CTGE setup used in $[1,8]$. The catalog sources are placed at their respective distances and their total flux is weighted according to the flux weight, both from [4]. The background sources are placed continuously following a source evolution $\propto(1+z)^{m}$, with either $m=0, m=5.0$ as expected for AGNs, or the star formation rate as given in [9] for SBGs ( $m=3.4$ for $z<0.97)$. For this first test of the method no cosmological expansion of the universe is considered.

From all arriving CRs we calculate probability distributions for each of the three observables, that we can compare with the data to find the best fit parameters, in a very similar way to [1]. For the energy, we bin the arriving CRs into 24 bins $\left(10^{18.0} \mathrm{eV}, 10^{18.1} \mathrm{eV} \ldots 10^{20.4} \mathrm{eV}\right)$. For $X_{\max }$, we calculate the expected probability distribution from the Gumbel distributions [10] as in [1] with the EPOSLHC hadronic interaction model, using $X_{\max }$ bins of width $20 \mathrm{~g} \mathrm{~cm}^{-2}$ and the same energy bins as for the spectrum up to $10^{19.6} \mathrm{eV}$ plus one combined energy bin above. For the arrival directions, we produce probability maps that would be expected for CRs deflected by local turbulent magnetic fields, where the deflection angle scales in proportion to the inverse of the rigidity. This is done by introducing Fisher distributions around each catalog source with a width $\delta_{S}=\delta_{0} Z_{\mathrm{det}} \frac{10 \mathrm{EeV}}{E_{\mathrm{det}}}$, using the representative elemental charges $Z_{\mathrm{det}}=1,2,7,14$ and 26 for $A_{\mathrm{det}} \in\{1\},\{2,3,4\},\{5, \ldots, 22\}$, $\{23, \ldots, 38\}$ and $\{39, \ldots, 56\}$ respectively. Each Fisher distribution is weighted by the observatory exposure in the arrival direction as well as the number of arriving events with the respective charge $Z_{\text {det }}$ in the energy bin $E_{\text {det }}$, which depends on the source weight and distance. Hence, the size of the Fisher distributions decreases as the rigidity increases with energy, and the widths and depths of the distributions contain information about the injected particles at the sources as well as the propagation distance and the size of the turbulent smearing. The background probability map follows the observatory exposure, as expected for an isotropic distribution of farther away background sources.

The background and catalog contributions are then combined into the total model of the measured observables, after weighting them with a signal fraction $f_{0}$. This signal fraction is defined for all events above $\log _{10}\left(E_{\mathrm{det}} / \mathrm{eV}\right)=18.7$ as this is the energy threshold that we will later use for 
the fit. For the probability maps, this leads to a combined pdf in each energy bin $e$, defined as:

$$
\operatorname{pdf}_{e}=f_{S}\left(f_{0}, E_{\mathrm{det}}^{e}\right) \cdot S\left(E_{\mathrm{det}}^{e}, \delta_{S}\left(E_{\mathrm{det}}^{e}\right)\right)+\left(1-f_{S}\left(f_{0}, E_{\mathrm{det}}^{e}\right)\right) \cdot B
$$

Here, the function $f_{S}\left(f_{0}, E_{\mathrm{det}}^{e}\right)$ describes the dependence of the catalog contribution on the energy. For close sources like the SBGs the catalog contribution increases with energy, as far away background sources do not contribute at the highest energies. We have now built a model of observed energies, energy-dependent depths of shower maximum as well as energy-dependent arrival direction probabilities which can be compared with the data to determine the model parameters.

\section{Fit method}

In total, the model has nine fit parameters: the spectral index $\gamma$, the maximum rigidity $R_{\text {cut }}$, four out of the five element fractions $a_{i},{ }^{1}$ the flux normalization $J_{0}$, the magnetic field blurring angle $\delta_{0}$ and the total signal fraction $f_{0}$. Additionally, it is possible to include systematic shifts of the energy and $X_{\max }$ scales as nuisance parameters $v$. The systematic uncertainty on the FD energy scale is $14 \%$ [12] and that for $X_{\max }$ is energy dependent, between $6 \mathrm{~g} \mathrm{~cm}^{-2}$ and $8 \mathrm{~g} \mathrm{~cm}^{-2}$ [11]. It was shown in $[1,8]$ that the hadronic interaction model can have a major impact on the fit results, and this can in part be parameterized by the shift of the $X_{\max }$ scale. We determine all fit parameters simultaneously via a Bayesian method using a Markov Chain Monte Carlo (MCMC) sampler, which samples the posterior distribution and hence enables us to determine the fit parameters as well as their uncertainties. The prior distributions are the same as in [1].2 Also, the likelihood function for the measured energy spectrum is modeled as a Poissonian and the $X_{\max }$ likelihood as a multinomial as in [1]. For the arrival directions, we use a similar likelihood function to eq. 1, only that we bin the events into the detected energy bins $j$ and use the modeled energy-dependent probability maps $\operatorname{pdf}_{j}$ instead of $\mathrm{pdf}_{\mathrm{AD}}$. As the three observables are independent measurements, the total likelihood function is given as a multiplication of the single likelihood functions.

We choose to only include $E_{\text {det }}$ bins above $\log _{10}\left(E_{\text {det }} / \mathrm{eV}\right)=19.0$ in the energy and the $X_{\max }$ likelihoods, as we expect contributions apart from our catalog sources below this energy, e.g. from Galactic or other lower-energy extragalactic source classes, which is supported by the findings of [8]. But, since we do not want our model to produce more low-energy particles below this threshold than are measured by Auger, we include the three energy bins with $\log _{10}\left(E_{\text {det }} / \mathrm{eV}\right)$ between 18.7 and 19.0 in the energy likelihood only if the model flux becomes higher than the measured one. For the arrival directions, we set an energy threshold at $\log _{10}\left(E_{\mathrm{det}} / \mathrm{eV}\right)=19.3$, as we do not intend to model the measured large-scale dipole [13] in the arrival probability maps.

\section{Benchmark simulations}

We demonstrate the method's sensitivity using simulations, which resemble the measured data in all three observables. For that, we apply the fit to the data of the Pierre Auger Observatory, using only the homogeneous background model $\left(f_{0}=0\right)$, which corresponds to the setup in [1] without

${ }^{1}$ The condition $\sum_{i} a_{i}=1$ is already incorporated.

${ }^{2}$ Gaussian priors for systematic uncertainties, uninformed prior for $J_{0}$, uniform bounded priors for other parameters. 
any contribution of the arrival directions observable. We use the $X_{\max }$ distributions from [11] and the SD energy spectrum from [12], including a forward folding method and detector effects on $X_{\max }$ as in [1]. The source evolution parameter is $m=3.4$. The best-fit parameters can be seen in table 1 . We find that a negative spectral index $\gamma$ describes the data best for this evolution, similar to what was also found in [1]. As one can see, the best-fit energy scale shift is 0 , as is also found in [8], and for speed reasons we will refrain from including it as a nuisance parameter in all following fits.

\begin{tabular}{ccccccccc}
$\gamma$ & $\log _{10}\left(R_{\mathrm{cut}} / V\right)$ & $a_{\mathrm{H}}$ & $a_{\mathrm{He}}$ & $a_{\mathrm{N}}$ & $a_{\mathrm{Si}}$ & $a_{\mathrm{Fe}}$ & $v_{\mathrm{X}_{\max }} / \sigma$ & $v_{\mathrm{E}} / \sigma$ \\
\hline-0.65 & 18.29 & $23 \%$ & $2 \%$ & $70 \%$ & $4.0 \%$ & $0.15 \%$ & -2.0 & 0.0
\end{tabular}

Table 1: Best-fit parameters used for benchmark simulation

To this simulation, we add a contribution from the SBG catalog with a signal fraction of only $f_{0}=1.2 \%$ above $10^{18.7} \mathrm{eV}$ and a smearing of $\delta_{0}=14.3^{\circ}$, which means a CR with rigidity $10 \mathrm{EV}$ is smeared by a Fisher distribution with this width. These parameters were chosen so that $\mathrm{TS}_{\mathrm{AD}}^{\mathrm{SBG}}$ approximately matches between the simulation and the measured data, as can be seen in fig. 1 (left). Even though the simulation parameters were not chosen to also get an agreement between $\mathrm{TS}_{\mathrm{AD}}^{\mathrm{AGN}}$ on the benchmark simulation and the data, they are still of the same order of magnitude. The arrival directions of all events with $E_{\text {det }} \geq 38 \mathrm{EeV}$ are visualized in fig. 1 (right), along with the locations of the strongest sources. As one can see in fig. 2, the energy spectrum and $X_{\max }$ distributions of the simulation still match the measured ones because of the small signal fraction. The dashed line shows the contribution of the catalog sources and it is visible that even though $f_{0}$ is so small, the contribution becomes significant $\left(f_{S}\left(f_{0}=1.2 \%, E_{\mathrm{det}} \geq 10^{20} \mathrm{eV}\right) \approx 40 \%\right)$ at the highest energies because the SBGs are close and their relative flux increases compared to the further away background sources. The number of events ejected at the source is adjusted, so that the flux on Earth matches the measured one from [12], as visible in fig. 2. Additionally, we adapt the number of events with $X_{\max }$ values in each energy bin to match data from [11], it is only a fraction of all events due to the limited FD duty cycle. We now have a simulation which resembles the measured data in all three observables and we can hence determine the expected sensitivity of the fit in a realistic scenario.

\section{Results and Discussion}

We apply different models to the benchmark simulation to determine if the fit is able to differentiate between the true $^{3}$ (SBGs) and false (AGNs) source classes and between the true source evolution (SFR evolution, $m \simeq 3.4$ ) and the false ones $(m=0.0, m=5.0)$. A visual representation of the best-fit results for the different models is shown in fig. 3. We give the confidence regions using the MCMC sampler for posterior sampling as well as the maximum a posteriori (MAP). The spectral parameters can be reconstructed well even with the reference models not including a catalog, although they get shifted for the different source evolutions. The clear correlation between $\gamma$ and $R_{\text {cut }}$ is visible, as also observed in [1]. Additionally, the signal fraction and the smearing are highly correlated, as also observed in [2]. The fit also determines a non-zero signal fraction for the AGN models, but the uncertainties are much larger. From the confidence regions, it becomes

\footnotetext{
${ }^{3}$ Here, true and false only refer to the simulated truth and do not have any connection to the real universe.
} 

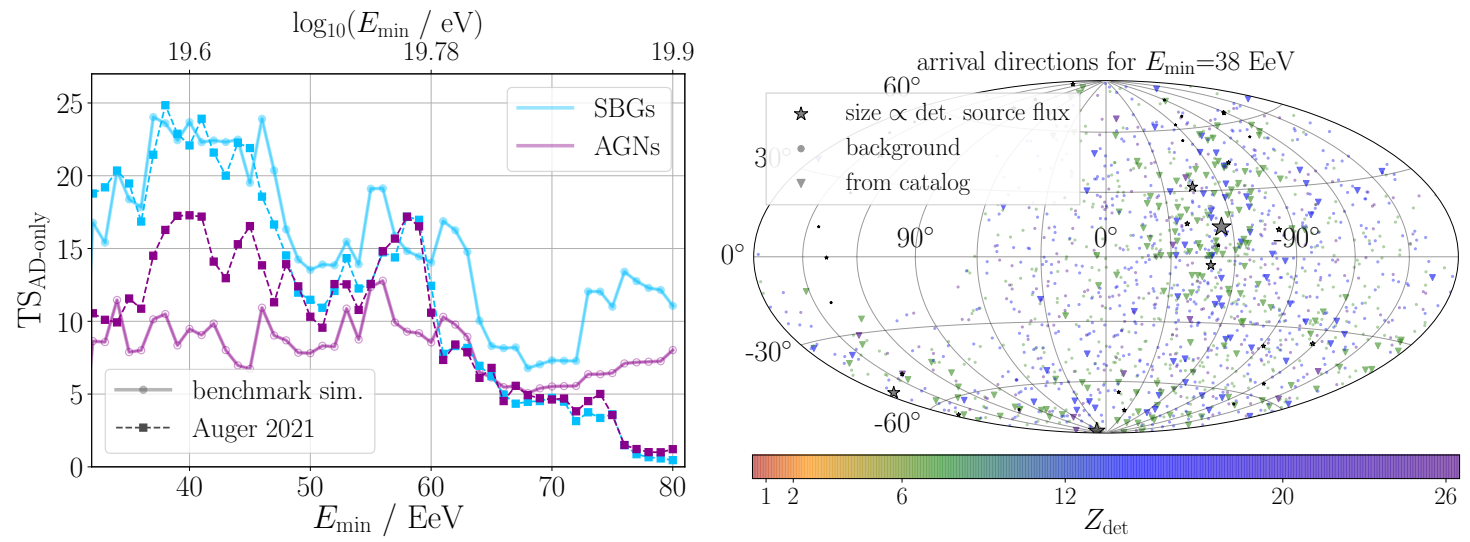

Figure 1: Test statistic of the arrival directions analysis (left) for the benchmark simulation as well as the data from [4]. A good agreement between both is visible for the AGN and SBG models. Arrival directions (right) for $E_{\mathrm{det}} \geq 38 \mathrm{EeV}$ for detected CRs of the benchmark simulation with charges $Z_{\mathrm{det}}$ as a colorbar.
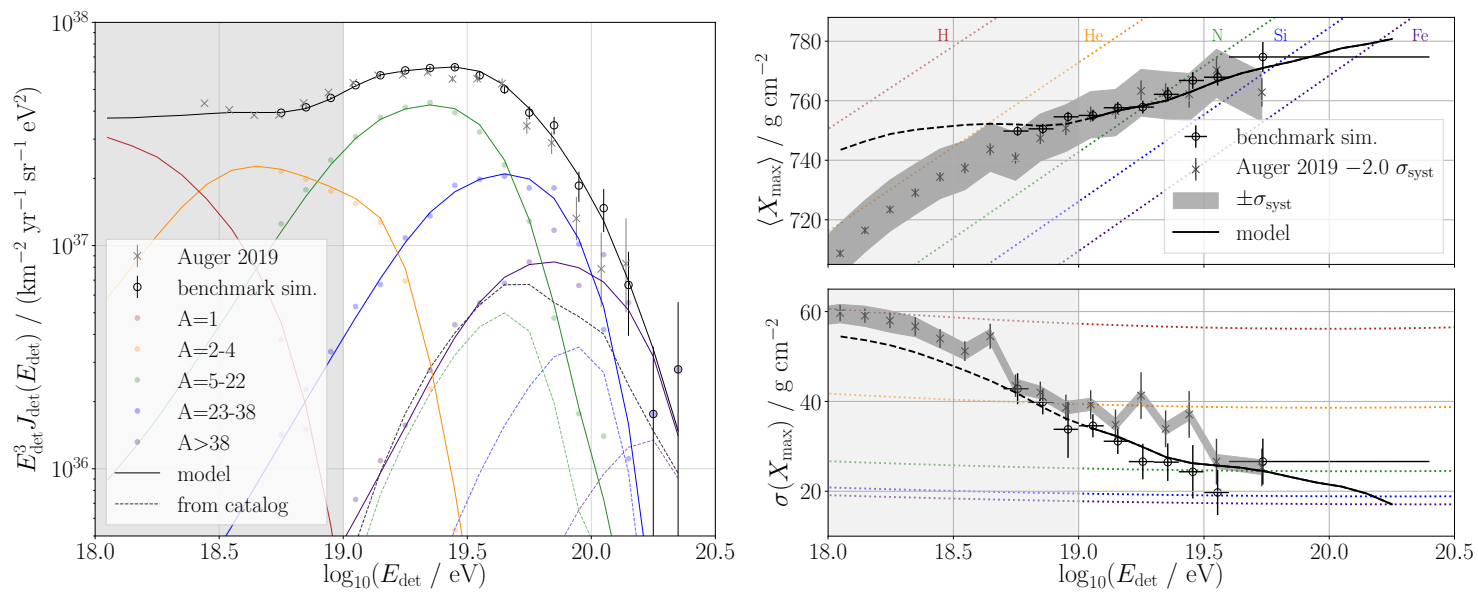

Figure 2: Energy spectrum (left) and mean (upper right), standard deviation (lower right) of the $X_{\max }$ distributions of the benchmark simulation, marked with round markers. The model, reweighted using the benchmark parameters, is shown as lines. The contribution by the SBG catalog is shown as dashed lines in the energy spectrum. For comparison, the data measured by the Pierre Auger Observatory [11, 12] is shown as cross markers, with $X_{\max }$ shifted by $-2 \sigma_{\text {syst }}$. The gray shaded area below $\log _{10}\left(E_{\text {det }}\right)=19.0$ is not part of the fit in the following, apart from the bins between $\log _{10}\left(E_{\text {det }}\right)=[18.7-19.0]$ for the spectrum, see text.

apparent that the primary Helium and Hydrogen fractions are basically unknown, which makes sense as the cutoff energies of these light particles turn out to be below the energy threshold. The heavier fractions are better constrained. From the best-fit parameters at the MAP we can calculate the $\log$-likelihood $\log \mathcal{L}$ ratio, multiplied with a factor 2 in analogy to eq. 2 [2], and the deviance ${ }^{4}$ for each model and thereby determine which model best describes the benchmark simulation. The results are given in table 2 . One can see that the spectrum deviance $D_{E}$ is very small, especially for the true source evolution of $m=3.4$. A good description of the data with a small $D$ is possible also for $m=0$, even though the spectrum parameters are not close to the truth, as shown in fig. 3 . The log-likelihood values demonstrate that the true model can be clearly identified to fit best. The AGN model cannot describe the energy-dependent arrival directions of the benchmark simulation

\footnotetext{
${ }^{4}$ The negative log-likelihood ratio of the model and the saturated model, which perfectly describes the data, see [1].
} 

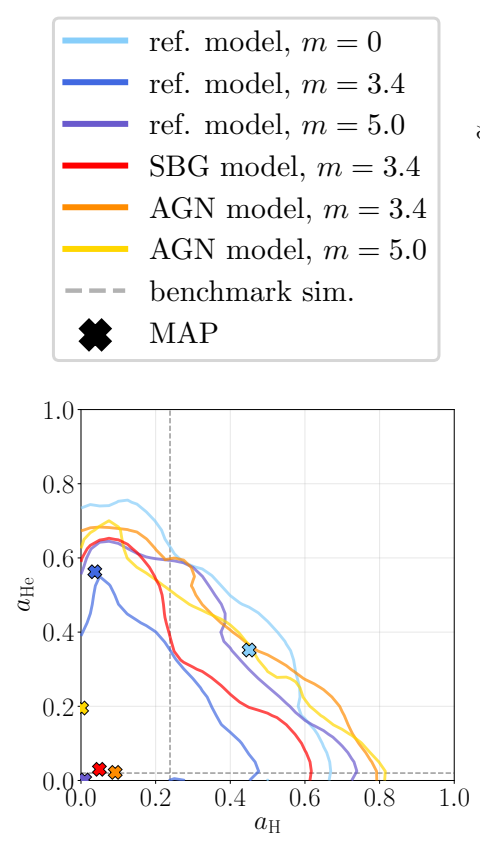
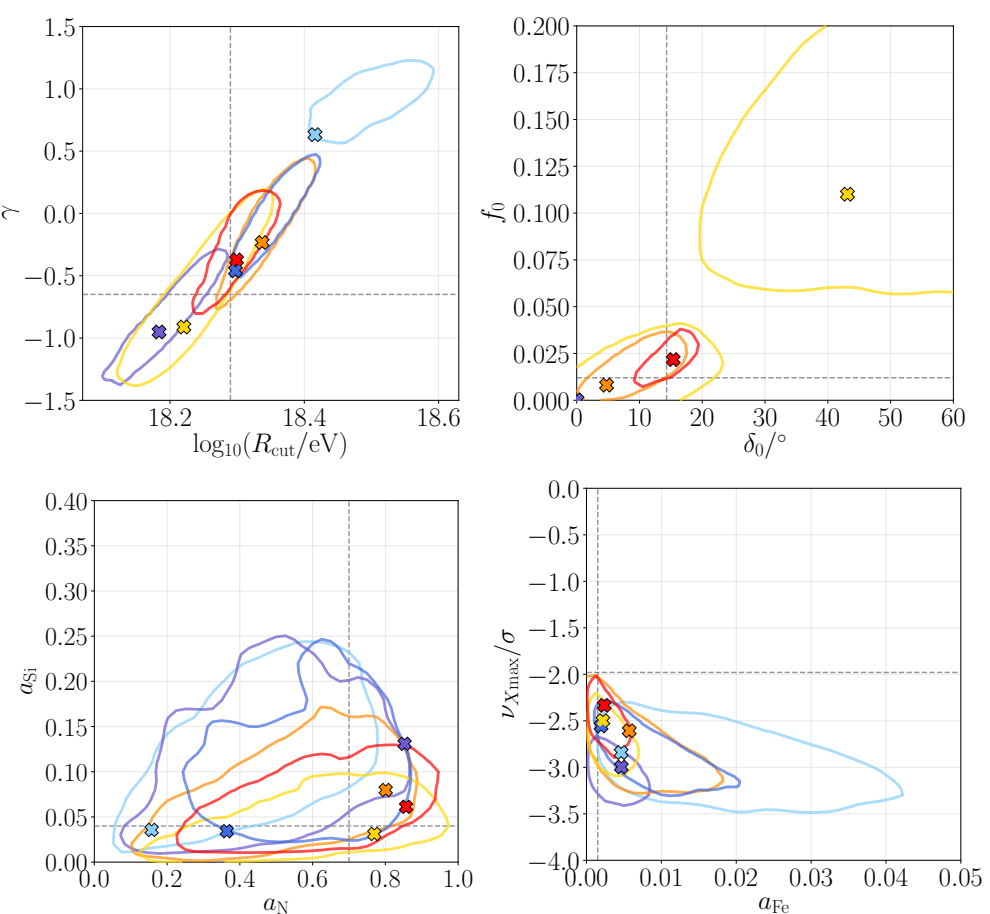

Figure 3: Best-fit parameters of the different models on the benchmark simulation, MAP and $90 \%$ confidence regions are shown. Gray dashed lines mark the benchmark parameters (see table 1). One can see that the parameters are reconstructed best for $m=3.4$ and the SBG model, the simulated truth.

as well as the SBG model, even when using the true source evolution $m=3.4$, which shows that using these as an additional observable is a powerful tool to distinguish different source catalogs. The expected sensitivity of the method and hence the conversion of the log-likelihood ratios to a $p$-value, calculated from isotropic simulations, is discussed in the following.

\begin{tabular}{l|rcc|cc} 
& $D_{E}$ & $D_{X_{\max }}$ & $D_{\text {total }}$ & $2 \log \frac{\mathcal{L}_{\mathrm{AD}}}{\mathcal{L}_{\mathrm{AD}}^{\text {ref } m=3.4}}$ & $2 \log \frac{\mathcal{L}_{\text {sum }}}{\mathcal{L}_{\text {sum }}^{\text {ref } m=3.4}}$ \\
\hline \hline ref. model $\left(f_{0}=0, m=0\right)$ & 6.0 & 82.1 & 88.1 & 0 & -0.8 \\
ref. model $\left(f_{0}=0, m=3.4\right)$ & 5.8 & 81.5 & 87.3 & 0 & 0 \\
ref. model $\left(f_{0}=0, m=5.0\right)$ & 12.9 & 84.0 & 96.9 & 0 & -9.6 \\
\hline SBG model $(m=3.4) \rightarrow$ sim. truth & 5.5 & 80.2 & 85.7 & 30.6 & 32.4 \\
AGN model $(m=3.4)$ & 6.0 & 81.8 & 87.8 & 11.2 & 10.8 \\
AGN model $(m=5.0)$ & 5.6 & 84.1 & 89.9 & 1.4 & -1.0
\end{tabular}

Table 2: Deviance $D$ and $\log$-likelihood ratio (normalized to reference model with $m=3.4$ ) at maximum a posterior (MAP) for different models on the benchmark simulation.

For estimating the sensitivity of the method, we compare the observed log-likelihood ratio $2 \log \left(\mathcal{L}_{\text {sum }}^{\mathrm{SBG}} / \mathcal{L}_{\text {sum }}^{\text {ref } m=3.4}\right)=32.4$ from the benchmark simulation to the expectation from isotropic simulations. These simulations contain the same source parameters as given in table 1, and only a homogeneous source distribution. In fig. 4, the log-likelihood ratios are displayed. One can see that the likelihood ratio observed on the benchmark simulation is exceptionally large compared to those from the isotropic simulations. The ratio can also be viewed separately for the three observables, even though only the total likelihood has been maximized. It is evident that the arrival directions are 

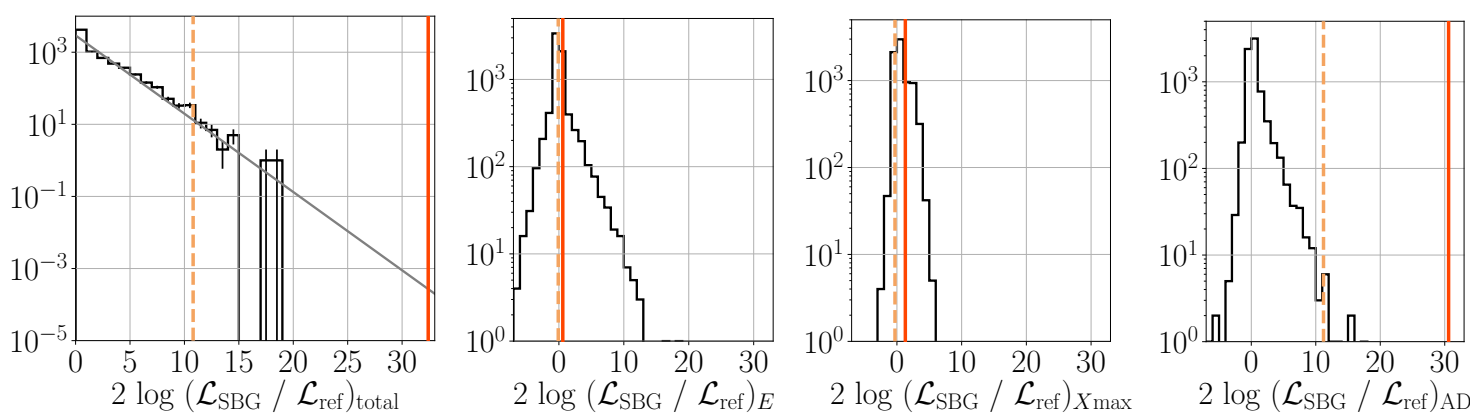

Figure 4: Log-likelihood ratio between SBG and reference model (compare table 2) on isotropic simulations (histogram) and on the benchmark simulation (red solid line). For comparison, the AGN ratio is also displayed (orange dashed line). The $\chi^{2}$ distribution with $n d f=2$ is shown on the left for the total ratio (gray line).

most important for the determination of the correct source catalog. Fig. 4 (left) also shows that at least the tail of the isotropic histogram is well described by a $\chi^{2}$ distribution with two degrees of freedom, as is expected because the catalog model has two more fit parameters $\left(f_{0}, \delta_{0}\right)$ than the reference model. From this, we can calculate $p$-values $p_{\mathrm{SBG}} \simeq 9 \cdot 10^{-8}$ and $p_{\mathrm{AGN}} \simeq 5 \cdot 10^{-3}$. These can be compared to the values for the arrival-directions only analysis [2] on the benchmark simulation (cf. fig. 1) which can also be calculated using the same $\chi^{2}$ distribution: $p_{\mathrm{SBG}}^{\mathrm{AD}-\text { only, pre-trial }} \simeq 6.1 \cdot 10^{-6}$, $p_{\mathrm{AGN}}^{\mathrm{AD}-\text { only, pre-trial }} \simeq 1.7 \cdot 10^{-3}$. The $p$-value for the false AGN model is approximately the same, while the energy-dependent fit including all observables noticeably increases the sensitivity for identifying the true SBG model on the benchmark simulation. Additionally, unlike [2] we do not need any scan of the energy threshold and hence no further penalization.

In summary, we have shown that the arrival direction observable can be included in the combined fit of energies and depth of shower maximum distributions, and that this enables us to determine the energy-dependent contribution of a source catalog to the flux of ultra-high-energy CRs at Earth. The fit including all three observables has a much better sensitivity to distinguish between the source catalogs of AGNs and SBGs.

\section{References}

[1] A. Aab et al. [Pierre Auger Coll.], J. Cosmol. Astropart. P. 04 (2017) 038, [1612.07155].

[2] A. Aab et al. [Pierre Auger Coll.], Astrophys. J. Lett. 853 (2018) L29, [1801. 06160].

[3] L. Caccianiga [for the Pierre Auger Coll.], Proc. 36th Int. Cosmic Ray Conf., Madison, US (2019), PoS(ICRC2019)206, [1909.09073].

[4] J. Biteau [for the Pierre Auger Coll.], these proceedings.

[5] R. Alves Batista et al., J. Cosmol. Astropart. P. 1605 (2016) 038, [1603.07142].

[6] R. Gilmore et al., Mon. Not. R. Astron. Soc. 422 (2012) 3189, [1104. 0671].

[7] A. Koning and D. Rochman, Nucl. Data Sheets 113 (2012) 2841.

[8] E. Guido [for the Pierre Auger Coll.], these proceedings.

[9] A. M. Hopkins et al., Astrophys. J. 651 (2006) 142, [astro-ph/0601463].

[10] M. De Domenico et al., J. Cosmol, Astropart. P. 1307 (2013) 050, [1305.2331].

[11] A. Yushkov [for the Pierre Auger Coll.], Proc. 36th Int. Cosmic Ray Conf., Madison, US (2019), PoS(ICRC2019)482, [1909.09073].

[12] V. Verzi [for the Pierre Auger Coll.], Proc. 36th Int. Cosmic Ray Conf., Madison, US (2019), PoS(ICRC2019)450, [1909.09073].

[13] A. Aab et al. [Pierre Auger Coll.], Science 357 (2017) 1266, [1709. 07321]. 


\section{The Pierre Auger Collaboration}

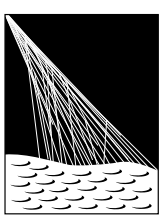

PIERRE

AUSGERVATORY

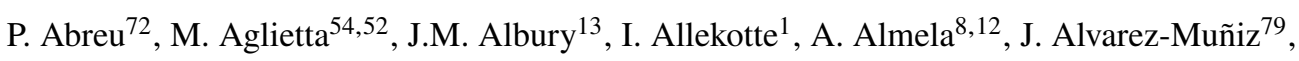
R. Alves Batista ${ }^{80}$, G.A. Anastasi ${ }^{63,52}$, L. Anchordoqui ${ }^{87}$, B. Andrada ${ }^{8}$, S. Andringa ${ }^{72}$, C. $\mathrm{Aramo}^{50}$, P.R. Araújo Ferreira ${ }^{42}$, J. C. Arteaga Velázquez ${ }^{67}$, H. Asorey $^{8}$, P. Assis ${ }^{72}$, G. Avila ${ }^{11}$, A.M. Badescu ${ }^{75}$, A. Bakalova ${ }^{32}$, A. Balaceanu ${ }^{73}$, F. Barbato ${ }^{45,46}$, R.J. Barreira Luz $^{72}$, K.H. Becker ${ }^{38}$, J.A. Bellido ${ }^{13,69}$, C. Berat ${ }^{36}$, M.E. Bertaina ${ }^{63,52}$, X. Bertou ${ }^{1}$, P.L. Biermann ${ }^{b}$, V. Binet ${ }^{6}$, K. Bismark ${ }^{39,8}$, T. Bister ${ }^{42}$, J. Biteau ${ }^{37}$, J. Blazek ${ }^{32}$, C. Bleve ${ }^{36}$, M. Boháčová ${ }^{32}$, D. Boncioli ${ }^{57,46}$, C. Bonifazi ${ }^{9,26}$, L. Bonneau Arbeletche ${ }^{21}$, N. Borodai ${ }^{70}$, A.M. Botti ${ }^{8}$, J. Brack ${ }^{d}$, T. Bretz ${ }^{42}$, P.G. Brichetto Orchera ${ }^{8}$, F.L. Briechle ${ }^{42}$, P. Buchholz ${ }^{44}$, A. Bueno ${ }^{78}$, S. Buitink ${ }^{15}$, M. Buscemi ${ }^{47}$, M. Büsken ${ }^{39,8}$, K.S. Caballero-Mora ${ }^{66}$, L. Caccianiga ${ }^{59,49}$, F. Canfora ${ }^{80,81}$, I. Caracas ${ }^{38}$, J.M. Carceller ${ }^{78}$, R. Caruso ${ }^{58,47}$, A. Castellina ${ }^{54,52}$, F. Catalani ${ }^{19}$, G. Cataldi ${ }^{48}$, L. Cazon ${ }^{72}$, M. Cerda ${ }^{10}$, J.A. Chinellato ${ }^{22}$, J. Chudoba ${ }^{32}$, L. Chytka ${ }^{33}$, R.W. Clay ${ }^{13}$, A.C. Cobos Ceruttii ${ }^{7}$, R. Colalillo ${ }^{60,50}$, A. Coleman ${ }^{93}$, M.R. Coluccia ${ }^{48}$, R. Conceição ${ }^{72}$, A. Condorelli ${ }^{45,46}$, G. Consolati ${ }^{49,55}$, F. Contreras ${ }^{11}$, F. Convenga ${ }^{56,48}$, D. Correia dos Santos $^{28}$, C.E. Covault ${ }^{85}$, S. Dasso ${ }^{5,3}$, K. Daumiller ${ }^{41}$, B.R. Dawson ${ }^{13}$, J.A. Day ${ }^{13}$, R.M. de Almeida $^{28}$, J. de Jesús ${ }^{8,41}$, S.J. de Jong ${ }^{80,81}$, G. De Mauro ${ }^{80,81}$, J.R.T. de Mello Neto ${ }^{26,27}$, I. De Mitri ${ }^{45,46}$, J. de Oliveira ${ }^{18}$, D. de Oliveira Franco ${ }^{22}$, F. de Palma ${ }^{56,48}$, V. de Souza $^{20}$, E. De Vito ${ }^{56,48}$, M. del Río ${ }^{11}$, O. Deligny ${ }^{34}$, L. Deval ${ }^{41,8}$, A. di Matteo $^{52}$, C. Dobrigkeit ${ }^{22}$, J.C. D’Olivo ${ }^{68}$, L.M. Domingues Mendes ${ }^{72}$, R.C. dos Anjos ${ }^{25}$, D. dos Santos $^{28}$, M.T. Dova ${ }^{4}$, J. Ebr ${ }^{32}$, R. Engel ${ }^{39,41}$, I. Epicoco ${ }^{56,48}$, M. Erdmann ${ }^{42}$, C.O. Escobar ${ }^{a}$, A. Etchegoyen ${ }^{8,12}$, H. Falcke ${ }^{80,82,81}$, J. Farmer ${ }^{92}$, G. Farrar ${ }^{90}$, A.C. Fauth ${ }^{22}$, N. Fazzini ${ }^{a}$, F. Feldbusch ${ }^{40}$, F. Fenu ${ }^{54,52}$,

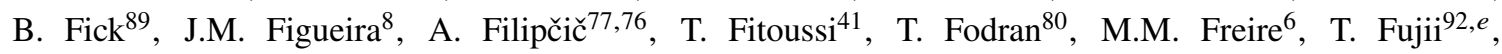
A. Fuster $^{8,12}$, C. Galea ${ }^{80}$, C. Galelli ${ }^{59,49}$, B. García ${ }^{7}$, A.L. Garcia Vegas ${ }^{42}$, H. Gemmeke ${ }^{40}$, F. Gesualdi ${ }^{8,41}$, A. Gherghel-Lascu ${ }^{73}$, P.L. Ghia ${ }^{34}$, U. Giaccari ${ }^{80}$, M. Giammarchi ${ }^{49}$, J. Glombitza ${ }^{42}$, F. Gobbi ${ }^{10}$, F. Gollan ${ }^{8}$, G. Golup ${ }^{1}$, M. Gómez Berisso ${ }^{1}$, P.F. Gómez Vitale ${ }^{11}$, J.P. Gongora ${ }^{11}$, J.M. González ${ }^{1}$, N. González ${ }^{14}$, I. Goos ${ }^{1,41}$, D. Góra ${ }^{70}$, A. Gorgi ${ }^{54,52}$, M. Gottowik ${ }^{38}$, T.D. Grubb ${ }^{13}$, F. Guarino ${ }^{60,50}$, G.P. Guedes ${ }^{23}$, E. Guido ${ }^{52,63}$, S. $\mathrm{Hahn}^{41,8}$, P. $\mathrm{Hamal}^{32}$, M.R. Hampel ${ }^{8}$, P. Hansen ${ }^{4}$, D. Harari ${ }^{1}$, V.M. Harvey ${ }^{13}$, A. Haungs ${ }^{41}$, T. Hebbeker ${ }^{42}$, D. Heck ${ }^{41}$, G.C. Hill ${ }^{13}$, C. Hojvat ${ }^{a}$, J.R. Hörandel ${ }^{80,81}$, P. Horvath ${ }^{33}$, M. Hrabovský ${ }^{33}$, T. Huege ${ }^{41,15}$, A. Insolia ${ }^{58,47}$, P.G. Isar $^{74}$, P. Janecek ${ }^{32}$, J.A. Johnsen ${ }^{86}$, J. Jurysek ${ }^{32}$, A. Kääpä ${ }^{38}$, K.H. Kampert ${ }^{38}$, N. Karastathis ${ }^{41}$, B. Keilhauer ${ }^{41}$, J. Kemp ${ }^{42}$, A. Khakurdikar ${ }^{80}$, V.V. Kizakke Covilakam $^{8,41}$, H.O. Klages ${ }^{41}$, M. Kleifges ${ }^{40}$, J. Kleinfeller ${ }^{10}$, M. Köpke ${ }^{39}$, N. Kunka ${ }^{40}$, B.L. Lago ${ }^{17}$, R.G. Lang ${ }^{20}$, N. Langner ${ }^{42}$, M.A. Leigui de Oliveira ${ }^{24}$, V. Lenok $^{41}$, A. Letessier-Selvon ${ }^{35}$, I. LhenryYvon $^{34}$, D. Lo Presti ${ }^{58,47}$, L. Lopes ${ }^{72}$, R. López ${ }^{64}$, L. Lu ${ }^{94}$, Q. Luce ${ }^{39}$, J.P. Lundquist ${ }^{76}$, A. Machado Payeras $^{22}$, G. Mancarella ${ }^{56,48}$, D. Mandat ${ }^{32}$, B.C. Manning ${ }^{13}$, J. Manshanden ${ }^{43}$, P. Mantsch ${ }^{a}$, S. Marafico ${ }^{34}$, A.G. Mariazzi ${ }^{4}$, I.C. Mariş ${ }^{14}$, G. Marsella ${ }^{61,47}$, D. Martello ${ }^{56,48}$, S. Martinelli ${ }^{41,8}$, O. Martínez Bravo ${ }^{64}$, M. Mastrodicasa ${ }^{57,46}$, H.J. Mathes ${ }^{41}$, J. Matthews ${ }^{88}$, G. Matthiae ${ }^{62,51}$, E. Mayotte ${ }^{38}$, P.O. Mazur ${ }^{a}$, G. MedinaTanco $^{68}$, D. Melo $^{8}$, A. Menshikov ${ }^{40}$, K.-D. Merenda ${ }^{86}$, S. Michal ${ }^{33}$, M.I. Micheletti ${ }^{6}$, L. Miramonti ${ }^{59,49}$, S. Mollerach ${ }^{1}$, F. Montanet ${ }^{36}$, C. Morello ${ }^{54,52}$, M. Mostafá ${ }^{91}$, A.L. Müller ${ }^{8}$, M.A. Muller ${ }^{22}$, K. Mulrey ${ }^{15}$, R. Mussa ${ }^{52}$, M. Muzio ${ }^{90}$, W.M. Namasaka ${ }^{38}$, A. Nasr-Esfahani ${ }^{38}$, L. Nellen ${ }^{68}$, M. Niculescu-Oglinzanu ${ }^{73}$, M. Niechciol ${ }^{44}$, D. Nitz ${ }^{89}$, D. Nosek ${ }^{31}$, V. Novotny ${ }^{31}$, L. Nožka ${ }^{33}$, A Nucita ${ }^{56,48}$, L.A. Núñez ${ }^{30}$, M. Palatka ${ }^{32}$, J. Pallotta ${ }^{2}$, P. Papenbreer ${ }^{38}$, G. Parente ${ }^{79}$, A. Parra ${ }^{64}$, J. Pawlowsky ${ }^{38}$, M. Pech ${ }^{32}$, F. Pedreira ${ }^{79}$, J. Pȩkala ${ }^{70}$, R. Pelayo ${ }^{65}$, J. Peña-Rodriguez ${ }^{30}$, E.E. Pereira Martins ${ }^{39,8}$, J. Perez Armand ${ }^{21}$, C. Pérez Bertolli $^{8,41}$, M. Perlin ${ }^{8,41}$, L. Perrone ${ }^{56,48}$, S. Petrera ${ }^{45,46}$, T. Pierog ${ }^{41}$, M. Pimenta ${ }^{72}$, V. Pirronello ${ }^{58,47}$, M. Platino ${ }^{8}$, B. Pont $^{80}$, M. Pothast ${ }^{81,80}$, P. Privitera ${ }^{92}$, M. Prouza ${ }^{32}$, A. Puyleart ${ }^{89}$, S. Querchfeld ${ }^{38}$, J. Rautenberg ${ }^{38}$, D. Ravignani ${ }^{8}$, M. Reininghaus ${ }^{41,8}$, J. Ridky ${ }^{32}$, F. Riehn ${ }^{72}$, M. Risse ${ }^{44}$, V. Rizi ${ }^{57,46}$, W. Rodrigues de Carvalho ${ }^{21}$, J. Rodriguez Rojo ${ }^{11}$, M.J. Roncoroni ${ }^{8}$, S. Rossoni ${ }^{43}$, M. Roth ${ }^{41}$, E. Roulet ${ }^{1}$, A.C. Rovero ${ }^{5}$, P. Ruehl ${ }^{44}$, A. Saftoiu ${ }^{73}$, F. Salamida ${ }^{57,46}$, H. Salazar ${ }^{64}$, G. Salina ${ }^{51}$, J.D. Sanabria Gomez ${ }^{30}$, F. Sánchez ${ }^{8}$, E.M. Santos ${ }^{21}$, E. Santos ${ }^{32}$, F. Sarazin ${ }^{86}$, R. Sarmento ${ }^{72}$, C. Sarmiento-Cano ${ }^{8}$, R. Sato ${ }^{11}$, 
P. Savina ${ }^{56,48,34,94}$, C.M. Schäfer ${ }^{41}$, V. Scherini ${ }^{56,48}$, H. Schieler ${ }^{41}$, M. Schimassek ${ }^{39,8}$, M. Schimp ${ }^{38}$, F. Schlüter ${ }^{41,8}$, D. Schmidt ${ }^{39}$, O. Scholten ${ }^{84,15}$, P. Schovánek ${ }^{32}$, F.G. Schröder ${ }^{93,41}$, S. Schröder ${ }^{38}$, J. Schulte ${ }^{42}$, S.J. Sciutto ${ }^{4}$, M. Scornavacche ${ }^{8,41}$, A. Segreto ${ }^{53,47}$, S. Sehgal ${ }^{38}$, R.C. Shellard ${ }^{16}$, G. Sigl ${ }^{43}$, G. Silli ${ }^{8,41}$, O. Sima ${ }^{73, f}$, R. Šmída ${ }^{92}$, P. Sommers ${ }^{91}$, J.F. Soriano ${ }^{87}$, J. Souchard ${ }^{36}$, R. Squartini ${ }^{10}$, M. Stadelmaier ${ }^{41,8}$, D. Stanca ${ }^{73}$, S. Stanič ${ }^{76}$, J. Stasielak ${ }^{70}$, P. Stassi ${ }^{36}$, A. Streich ${ }^{39,8}$, M. Suárez-Durán ${ }^{14}$, T. Sudholz ${ }^{13}$, T. Suomijärvi ${ }^{37}$, A.D. Supanitsky ${ }^{8}$, Z. Szadkowski ${ }^{71}$, A. Tapia ${ }^{29}$, C. Taricco ${ }^{63,52}$, C. Timmermans ${ }^{81,80}$, O. Tkachenko ${ }^{41}$, P. Tobiska ${ }^{32}$, C.J. Todero Peixoto ${ }^{19}$, B. Tomé ${ }^{72}$, Z. Torrès ${ }^{36}$, A. Travaini ${ }^{10}$, P. Travnicek $^{32}$, C. Trimarelli ${ }^{57,46}$, M. Tueros ${ }^{4}$, R. Ulrich ${ }^{41}$, M. Unger ${ }^{41}$, L. Vaclavek ${ }^{33}$, M. Vacula ${ }^{33}$, J.F. Valdés Galicia ${ }^{68}$, L. Valore ${ }^{60,50}$, E. Varela ${ }^{64}$, A. Vásquez-Ramírez ${ }^{30}$, D. Veberič ${ }^{41}$, C. Ventura ${ }^{27}$, I.D. Vergara Quispe ${ }^{4}$, V. Verzi ${ }^{51}$, J. Vicha ${ }^{32}$, J. Vink $^{83}$, S. Vorobiov ${ }^{76}$, H. Wahlberg ${ }^{4}$, C. Watanabe ${ }^{26}$, A.A. Watson ${ }^{c}$, M. Weber ${ }^{40}$, A. Weindl ${ }^{41}$, L. Wiencke ${ }^{86}$, H. Wilczyński ${ }^{70}$, M. Wirtz ${ }^{42}$, D. Wittkowski ${ }^{38}$, B. Wundheiler ${ }^{8}$, A. Yushkov $^{32}$, O. Zapparrata ${ }^{14}$, E. Zas ${ }^{79}$, D. Zavrtanik ${ }^{76,77}$, M. Zavrtanik ${ }^{77,76}$, L. Zehrer ${ }^{76}$

${ }^{1}$ Centro Atómico Bariloche and Instituto Balseiro (CNEA-UNCuyo-CONICET), San Carlos de Bariloche, Argentina

${ }^{2}$ Centro de Investigaciones en Láseres y Aplicaciones, CITEDEF and CONICET, Villa Martelli, Argentina

${ }^{3}$ Departamento de Física and Departamento de Ciencias de la Atmósfera y los Océanos, FCEyN, Universidad de Buenos Aires and CONICET, Buenos Aires, Argentina

${ }^{4}$ IFLP, Universidad Nacional de La Plata and CONICET, La Plata, Argentina

${ }^{5}$ Instituto de Astronomía y Física del Espacio (IAFE, CONICET-UBA), Buenos Aires, Argentina

${ }^{6}$ Instituto de Física de Rosario (IFIR) - CONICET/U.N.R. and Facultad de Ciencias Bioquímicas y Farmacéuticas U.N.R., Rosario, Argentina

${ }^{7}$ Instituto de Tecnologías en Detección y Astropartículas (CNEA, CONICET, UNSAM), and Universidad Tecnológica Nacional - Facultad Regional Mendoza (CONICET/CNEA), Mendoza, Argentina

${ }^{8}$ Instituto de Tecnologías en Detección y Astropartículas (CNEA, CONICET, UNSAM), Buenos Aires, Argentina

${ }^{9}$ International Center of Advanced Studies and Instituto de Ciencias Físicas, ECyT-UNSAM and CONICET, Campus Miguelete - San Martín, Buenos Aires, Argentina

${ }^{10}$ Observatorio Pierre Auger, Malargüe, Argentina

${ }^{11}$ Observatorio Pierre Auger and Comisión Nacional de Energía Atómica, Malargüe, Argentina

12 Universidad Tecnológica Nacional - Facultad Regional Buenos Aires, Buenos Aires, Argentina

${ }^{13}$ University of Adelaide, Adelaide, S.A., Australia

14 Université Libre de Bruxelles (ULB), Brussels, Belgium

15 Vrije Universiteit Brussels, Brussels, Belgium

${ }^{16}$ Centro Brasileiro de Pesquisas Fisicas, Rio de Janeiro, RJ, Brazil

${ }^{17}$ Centro Federal de Educação Tecnológica Celso Suckow da Fonseca, Nova Friburgo, Brazil

${ }^{18}$ Instituto Federal de Educação, Ciência e Tecnologia do Rio de Janeiro (IFRJ), Brazil

${ }^{19}$ Universidade de São Paulo, Escola de Engenharia de Lorena, Lorena, SP, Brazil

${ }^{20}$ Universidade de São Paulo, Instituto de Física de São Carlos, São Carlos, SP, Brazil

${ }^{21}$ Universidade de São Paulo, Instituto de Física, São Paulo, SP, Brazil

${ }^{22}$ Universidade Estadual de Campinas, IFGW, Campinas, SP, Brazil

${ }^{23}$ Universidade Estadual de Feira de Santana, Feira de Santana, Brazil

${ }^{24}$ Universidade Federal do ABC, Santo André, SP, Brazil

${ }^{25}$ Universidade Federal do Paraná, Setor Palotina, Palotina, Brazil

${ }^{26}$ Universidade Federal do Rio de Janeiro, Instituto de Física, Rio de Janeiro, RJ, Brazil

${ }^{27}$ Universidade Federal do Rio de Janeiro (UFRJ), Observatório do Valongo, Rio de Janeiro, RJ, Brazil

${ }^{28}$ Universidade Federal Fluminense, EEIMVR, Volta Redonda, RJ, Brazil

${ }^{29}$ Universidad de Medellín, Medellín, Colombia

${ }^{30}$ Universidad Industrial de Santander, Bucaramanga, Colombia

${ }^{31}$ Charles University, Faculty of Mathematics and Physics, Institute of Particle and Nuclear Physics, Prague, Czech Republic

32 Institute of Physics of the Czech Academy of Sciences, Prague, Czech Republic 
${ }^{33}$ Palacky University, RCPTM, Olomouc, Czech Republic

34 CNRS/IN2P3, IJCLab, Université Paris-Saclay, Orsay, France

${ }^{35}$ Laboratoire de Physique Nucléaire et de Hautes Energies (LPNHE), Sorbonne Université, Université de Paris, CNRSIN2P3, Paris, France

${ }^{36}$ Univ. Grenoble Alpes, CNRS, Grenoble Institute of Engineering Univ. Grenoble Alpes, LPSC-IN2P3, 38000 Grenoble, France

${ }^{37}$ Université Paris-Saclay, CNRS/IN2P3, IJCLab, Orsay, France

38 Bergische Universität Wuppertal, Department of Physics, Wuppertal, Germany

${ }^{39}$ Karlsruhe Institute of Technology (KIT), Institute for Experimental Particle Physics, Karlsruhe, Germany

${ }^{40}$ Karlsruhe Institute of Technology (KIT), Institut für Prozessdatenverarbeitung und Elektronik, Karlsruhe, Germany

${ }^{41}$ Karlsruhe Institute of Technology (KIT), Institute for Astroparticle Physics, Karlsruhe, Germany

${ }^{42}$ RWTH Aachen University, III. Physikalisches Institut A, Aachen, Germany

43 Universität Hamburg, II. Institut für Theoretische Physik, Hamburg, Germany

${ }^{44}$ Universität Siegen, Department Physik - Experimentelle Teilchenphysik, Siegen, Germany

${ }^{45}$ Gran Sasso Science Institute, L'Aquila, Italy

46 INFN Laboratori Nazionali del Gran Sasso, Assergi (L'Aquila), Italy

${ }^{47}$ INFN, Sezione di Catania, Catania, Italy

${ }^{48}$ INFN, Sezione di Lecce, Lecce, Italy

${ }^{49}$ INFN, Sezione di Milano, Milano, Italy

${ }^{50}$ INFN, Sezione di Napoli, Napoli, Italy

${ }^{51}$ INFN, Sezione di Roma "Tor Vergata", Roma, Italy

52 INFN, Sezione di Torino, Torino, Italy

53 Istituto di Astrofisica Spaziale e Fisica Cosmica di Palermo (INAF), Palermo, Italy

54 Osservatorio Astrofisico di Torino (INAF), Torino, Italy

55 Politecnico di Milano, Dipartimento di Scienze e Tecnologie Aerospaziali, Milano, Italy

${ }^{56}$ Università del Salento, Dipartimento di Matematica e Fisica "E. De Giorgi”, Lecce, Italy

57 Università dell'Aquila, Dipartimento di Scienze Fisiche e Chimiche, L'Aquila, Italy

58 Università di Catania, Dipartimento di Fisica e Astronomia, Catania, Italy

${ }^{59}$ Università di Milano, Dipartimento di Fisica, Milano, Italy

${ }^{60}$ Università di Napoli "Federico II", Dipartimento di Fisica "Ettore Pancini”, Napoli, Italy

${ }^{61}$ Università di Palermo, Dipartimento di Fisica e Chimica "E. Segrè", Palermo, Italy

62 Università di Roma "Tor Vergata", Dipartimento di Fisica, Roma, Italy

${ }^{63}$ Università Torino, Dipartimento di Fisica, Torino, Italy

${ }^{64}$ Benemérita Universidad Autónoma de Puebla, Puebla, México

${ }^{65}$ Unidad Profesional Interdisciplinaria en Ingeniería y Tecnologías Avanzadas del Instituto Politécnico Nacional (UPIITA-IPN), México, D.F., México

66 Universidad Autónoma de Chiapas, Tuxtla Gutiérrez, Chiapas, México

${ }^{67}$ Universidad Michoacana de San Nicolás de Hidalgo, Morelia, Michoacán, México

${ }^{68}$ Universidad Nacional Autónoma de México, México, D.F., México

${ }^{69}$ Universidad Nacional de San Agustin de Arequipa, Facultad de Ciencias Naturales y Formales, Arequipa, Peru

${ }^{70}$ Institute of Nuclear Physics PAN, Krakow, Poland

${ }^{71}$ University of Łódź, Faculty of High-Energy Astrophysics, Łódź, Poland

${ }^{72}$ Laboratório de Instrumentação e Física Experimental de Partículas - LIP and Instituto Superior Técnico - IST, Universidade de Lisboa - UL, Lisboa, Portugal

73 "Horia Hulubei” National Institute for Physics and Nuclear Engineering, Bucharest-Magurele, Romania

${ }^{74}$ Institute of Space Science, Bucharest-Magurele, Romania

75 University Politehnica of Bucharest, Bucharest, Romania

76 Center for Astrophysics and Cosmology (CAC), University of Nova Gorica, Nova Gorica, Slovenia

${ }^{77}$ Experimental Particle Physics Department, J. Stefan Institute, Ljubljana, Slovenia

78 Universidad de Granada and C.A.F.P.E., Granada, Spain

${ }^{79}$ Instituto Galego de Física de Altas Enerxías (IGFAE), Universidade de Santiago de Compostela, Santiago de Compostela, Spain 
${ }^{80}$ IMAPP, Radboud University Nijmegen, Nijmegen, The Netherlands

${ }^{81}$ Nationaal Instituut voor Kernfysica en Hoge Energie Fysica (NIKHEF), Science Park, Amsterdam, The Netherlands

${ }^{82}$ Stichting Astronomisch Onderzoek in Nederland (ASTRON), Dwingeloo, The Netherlands

${ }^{83}$ Universiteit van Amsterdam, Faculty of Science, Amsterdam, The Netherlands

${ }^{84}$ University of Groningen, Kapteyn Astronomical Institute, Groningen, The Netherlands

85 Case Western Reserve University, Cleveland, OH, USA

86 Colorado School of Mines, Golden, CO, USA

${ }^{87}$ Department of Physics and Astronomy, Lehman College, City University of New York, Bronx, NY, USA

${ }^{88}$ Louisiana State University, Baton Rouge, LA, USA

${ }^{89}$ Michigan Technological University, Houghton, MI, USA

${ }^{90}$ New York University, New York, NY, USA

${ }^{91}$ Pennsylvania State University, University Park, PA, USA

92 University of Chicago, Enrico Fermi Institute, Chicago, IL, USA

93 University of Delaware, Department of Physics and Astronomy, Bartol Research Institute, Newark, DE, USA

94 University of Wisconsin-Madison, Department of Physics and WIPAC, Madison, WI, USA

${ }^{a}$ Fermi National Accelerator Laboratory, Fermilab, Batavia, IL, USA

${ }^{b}$ Max-Planck-Institut für Radioastronomie, Bonn, Germany

${ }^{c}$ School of Physics and Astronomy, University of Leeds, Leeds, United Kingdom

${ }^{d}$ Colorado State University, Fort Collins, CO, USA

$e^{e}$ now at Hakubi Center for Advanced Research and Graduate School of Science, Kyoto University, Kyoto, Japan

$f$ also at University of Bucharest, Physics Department, Bucharest, Romania 